\title{
The Effects of Omega3 on Oocyte Maturation
}

\author{
Ghorbani Vahed M, Maghzi P and Karimi Ghezeli Z
}

\begin{abstract}
It has been shown that fatty acids have role in oocyte maturation. The aim of this study was to determine the effects of omega3 on oocyte maturation in vitro. In our study, incubation of mouse oocytes with omega3 resulted in increased oocyte maturation. Our results indicated that omega3 play a role in oocyte maturation.
\end{abstract}

Keywords - Oocyte, Maturation, Omega3.

\section{INTRODUCTION}

Omega-3 fatty acids - also called $\omega-3$ fatty acids or n-3 fatty acids are polyunsaturated fatty acids (PUFAs) with a double bond $(\mathrm{C}=\mathrm{C})$ at the third carbon atom from the end of the carbon chain. The fatty acids have two ends, the carboxylic acid ($\mathrm{COOH}$ ) end, which is considered the beginning of the chain, thus "alpha", and the methyl (-CH3) end, which is considered the "tail" of the chain, thus "omega"; the double bond is at omega minus 3 (not dash 3). Common sources of plant oils containing the omega-3 ALA fatty acid include walnut, edible seeds, clary sage seed oil, algal oil, flaxseed oil, Sacha Inchi oil, Echium oil, and hemp oil, while sources of animal omega-3 EPA and DHA fatty acids include fish, fish oils, eggs from chickens fed EPA and DHA, squid oils, and krill oil.[1] Fatty acids improve the circulation to the genitals, which help support the prostate and the other element needed for reproduction.

It helps lower blood pressure, which helps with erectile dysfunction. Omega $3 \mathrm{~s}$ are important to the production of sperm.[2]

Flax oil and fish oil are rich in Omega-3 fatty acids. Omega-3 acids have been shown to help fertility by helping to regulate hormones in the body, increase cervical mucous, promote ovulation and overall improve the quality of the uterus by increasing the blood flow to the reproductive organs. Omega-3 fats also contain two acids that are crucial to good health: DHA and EPA. These two acids have been shown to help

Masoomeh Ghorbani Vahed (PhD-Fellow) (*corresponding author) is with the Department of Biology, Faculty of Basic Sciences, Islamic Azad University, Shiraz Branch, Shiraz, Iran. E-mail: m.ghorbani@yahoo.com

Pegah Maghzi (corresponding author) is with Department of Medical Surgical Nursing, Faculty of Nursing, Isfahan University of Medical Science, Isfahan, Iran. E-mail: Pegah.m.n2@gmail.com

Zahra Karimi Ghezeli is with Department of Pharmaceutical Chemistry, Faculty of Pharmaceutical Chemistry, Pharmaceutical Branch, Islamic Azad University, Tehran, Iran (IAUPS). E-mail: sandrakarimi20@gmail.com many forms of disease. Low levels of DHA have been linked to depression and other mental health issues. During pregnancy, a lack of DHA may be associated with premature birth, low birth weight and hyperactivity in children. [3]

Oocyte intracellular lipids are mainly stored in lipid droplets (LD) providing energy for proper growth and development. Lipids are also important signalling molecules involved in the regulatory mechanisms of maturation and hence in oocyte competence acquisition. Recent studies show that $\mathrm{LD}$ are highly dynamic organelles. They change their shape, volume, and location within the ooplasm as well as their interaction with other organelles during the maturation process. The droplets high lipid content has been correlated with impaired oocyte developmental competence and low cryosurvival. Yet the underlying mechanisms are not fully understood. In particular, the lipid-rich pig oocyte might be an excellent model to understand the role of lipids and fatty acid metabolism during the mammalian oocyte maturation and their implications on subsequent monospermic fertilization and preimplantation embryo development. [4].

\section{MATERIAL AND METHODS}

Oocytes were removed from mouse ovaries and cultured in cell culture media and incubated with different doses of omega3 and subsequently were studied through microscope and the maturation of oocytes were examined and compared with control and sham groups.

\section{RESULTS}

Our results indicated that exposure to 10 micrograms of omega3 led to significant increase in oocyte maturity compared to control cells $(\mathrm{P}<0.01)$. There was non-significant difference in oocyte maturation in sham compared to control group (Figure I) Fig. 1 shown in appendix.

\section{DISCUSSION}

In our study we found that omega3 has improving effect on oocyte maturation and so on reproductive system. Generally, the results indicated that healthy diets rich in some nutrients such as omega-3 fatty acids, some antioxidants (vitamin E, vitamin $\mathrm{C}, \beta$-carotene, selenium, zinc, cryptoxanthin and lycopene), other vitamins (vitamin D and folate) and low in saturated fatty acids and trans-fatty acids were as sociated with improved reproductive system function.[5] Supplements that have demonstrated positive effects on fertility include aescin, coenzyme Q 10 , glutathione, Korean red ginseng, L-carnitine, 
nigella sativa, omega-3, selenium, a combination of zinc and folate, and the Menevit antioxidant. [6] Studies also show that dietary omega3 has positive effects on treatment of some aspects of reproductive system disorders. [7] There is strong evidence linking consumption of diets high in omega-3 with reduced circulating peripheral inflammatory markers such as PGF(2 $\alpha$ ). Inflammatory eicosanoids including PGF(2 $\alpha)$, in particular, can significantly affect reproduction outcomes such as the onset of oestrus, embryo survival and parturition. [8] Omega- 6 and omega- 3 polyunsaturated fatty acids are reported to alter follicular growth, steroid synthesis and prostaglandin metabolism in the ovary and endometrium, respectively. Omega-6 fatty acids are believed to have pro-inflammatory and thus PGF $2 \alpha$-stimulating properties rendering them extra value as 'nutraceutical' early post-partum, while omega-3 fatty acids can weaken this inflammatory potency, leading to a higher chance of survival of the embryo when supplemented during the periconceptual period.[9] Beside the ovary, the fat body is an important organ involved in the metabolism and storage of the glycoproteins and lipids that will be transformed into the lipoglycoproteins of the yolk platelets in the ovary. [10] In non-human primates $\mathrm{n}-3$ fatty acid insufficiency during perinatal development leads to widespread deficits in functional connectivity in adult frontal cortical networks compared to primates raised on DHA-fortified diet. [11] The long chain omega-3 fatty acid also play important role in maturation of various tissues including brain tissue. [12].

\section{CONCLUSION}

According to our finding, omega 3 play a role in oocyte maturation.

\section{APPENDIX}

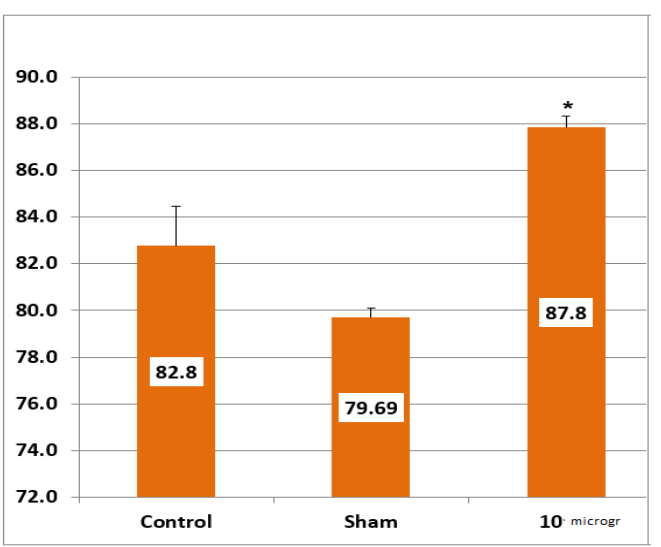

Figure I. Maturation of oocytes compared to control group.

* indicates significant increase compared to control group $(\mathrm{P}<0.01)$

\section{ACKNOWLEDGMENT}

We appreciate all who helped us to exert this study.

\section{REFERENCES}

[1] Sushchik NN Role of essential fatty acids in trophometabolic interactions in the freshwater ecosystems. Zhurnal Obshchei Biologii 2008, 69(4):299-316

[2] Rombaldi Bernardi J, de Souza Escobar R, Ferreira CF, Pelufo Silveira P.Fetal and neonatal levels of omega-3: effects on neurodevelopment, nutrition, and growth. ScientificWorldJournal. 2012;2012:202473.

[3] Sandra L. Huffman,Rajwinder K. Harika,Ans Eilander, Saskia J.M. Osendarp. Essential fats: how do they affect growth and development of infants and young children in developing countries? A literature review. 2011: 7(3), 44-65

[4] E. G. Prates, J. T. Nunes, R. M. PereiraA Role of Lipid Metabolism during Cumulus-Oocyte Complex Maturation: Impact of Lipid Modulators to Improve Embryo Production Mediators of Inflammation. 2014 (2014), Article ID 692067,

[5] Salas-Huetos A .Bulló M, Salas-Salvadó J. Dietary patterns, foods and nutrients in male fertility parameters and fecundability: a systematic review of observational studies. Hum Reprod Update. 2017;23(4):371-389.

[6] Yao DF, Mills JN.Male infertility: lifestyle factors and holistic, complementary, and alternative therapies. Asian J Androl. 2016 :18(3):410-8

[7] Rondanelli M, Perna S, Faliva M, Monteferrario F, Repaci E, Allieri F.Focus on metabolic and nutritional correlates of polycystic ovary syndrome and update on nutritional management of these critical phenomena. Arch Gynecol Obstet. 2014;290(6):1079-92

[8] Gulliver CE, Friend MA, King BJ, Clayton EH.The role of omega-3 polyunsaturated fatty acids in reproduction of sheep and cattle. Anim Reprod Sci. 2012;131(1-2):9-22.

[9] Leroy JL, Sturmey RG, Van Hoeck V, De Bie J, McKeegan PJ, Bols PEDietary fat supplementation and the consequences for oocyte and embryo quality: hype or significant benefit for dairy cow reproduction? Reprod Domest Anim. 2014;49(3):353-61

[10] Cuzin-Roudy J.Reproduction in Northern krill (Meganyctiphanes norvegica Sars). Adv Mar Biol. 2010;57:199-230

[11] McNamara RK, Vannest JJ, Valentine CJ.Role of perinatal longchain omega-3 fatty acids in cortical circuit maturation: Mechanisms and implications for psychopathology. World $\mathrm{J}$ Psychiatry. 2015 22;5(1):15-34

[12] Kuratko CN, Barrett EC, Nelson EB, Salem N Jr.The relationship of docosahexaenoic acid (DHA) with learning and behavior in healthy children: a review.Nutrients. 2013 Jul 19;5(7):2777-810. 